\title{
Review
}

\section{Translational toxicology and rescue strategies of the hERG channel dysfunction: biochemical and molecular mechanistic aspects}

\author{
Kai-ping ZHANG ${ }^{1,2}$, Bao-feng YANG ${ }^{1,2}$, Bao-xin $\mathrm{LI}^{1,2, *}$ \\ ${ }^{1}$ Department of Pharmacology, Harbin Medical University, Harbin, China; ${ }^{2}$ The State-Province Key Laboratories of Biomedicine- \\ Pharmaceutics of China (Key Laboratory of Cardiovascular Research, Ministry of Education), China
}

The human ether-à-go-go related gene (hERG) potassium channel is an obligatory anti-target for drug development on account of its essential role in cardiac repolarization and its close association with arrhythmia. Diverse drugs have been removed from the market owing to their inhibitory activity on the hERG channel and their contribution to acquired long QT syndrome (LQTS). Moreover, mutations that cause hERG channel dysfunction may induce congenital LQTS. Recently, an increasing number of biochemical and molecular mechanisms underlying hERG-associated LQTS have been reported. In fact, numerous potential biochemical and molecular rescue strategies are hidden within the biogenesis and regulating network. So far, rescue strategies of hERG channel dysfunction and LQTS mainly include activators, blockers, and molecules that interfere with specific links and other mechanisms. The aim of this review is to discuss the rescue strategies based on hERG channel toxicology from the biochemical and molecular perspectives.

Keywords: potassium channel; human ether-à-go-go related gene (hERG); long QT syndrome (LQTS); activator; blocker; siRNA; biogenesis; protein trafficking

Acta Pharmacologica Sinica(2014) 35: 1473-1484; doi: 10.1038/aps.2014.101; published online 24 Nov 2014

\section{Introduction}

The human ether-à-go-go related gene (hERG) potassium channel, also known as Kv11.1, is encoded by the KCNH2 gene located on chromosome $7 \mathrm{q} 36.1^{[1]}$. The main isoform of the hERG protein contains 1159 amino acids comprising 6 transmembrane segments, a Per-Arnt-Sim (PAS) domain at the $\mathrm{N}$ terminus and a cyclic-nucleotide-binding domain (cNBD) at the $C$ terminus ${ }^{[1,2]}$. The potassium current conducted by the hERG $\left(\mathrm{I}_{[\mathrm{Kr}]}\right)$ is essential for repolarization of cardiomyocytes after a heartbeat. Reduction of $\mathrm{I}_{[\mathrm{Kr}]}$ can lead to long QT syndrome (LQTS), which is characterized on the electrocardiogram by prolonged QT intervals and arrhythmia. In fact, hERG plays a role in various physiologic and pathologic processes such as cancer, digestive, secretory and reproductive systems, signaling, diseases of the nervous system as well as development ${ }^{[3]}$. However, research on hERG has focused mainly on cardiac repolarization and $\mathrm{LQTS}^{[3]}$.

LQTS may have severe manifestations including torsade de pointes $(\mathrm{TdP})$, syncope, and sudden cardiac death ${ }^{[4,5]}$. Type

\footnotetext{
* To whom correspondence should be addressed.

E-mail libx64@hotmail.com

Received 2014-05-14 Accepted 2014-08-20
}

2 LQTS (LQT2) refers specifically to a subclass of the disease that is due to genetic mutations in hERG that underlie approximately $45 \%$ of all congenital cases ${ }^{[1,4]}$. Prolongation of the QT interval may also result from medications that inhibit hERG channels as a side effect of the therapeutic action. Therefore, the United States Food and Drug Administration (FDA) has designated hERG as an obligatory anti-target in drug development. Existing drugs may also be withdrawn from clinical use because of their adverse effects on the hERG channel. Wellknown examples include terfenadine, cisapride, and astemizole.

For decades, the majority of studies in hERG toxicology have focused on the inhibitory effects of various compounds on hERG channel gating. However, several puzzles remain unsolved when applying the channel blockade theory. For example, arsenic trioxide, a drug used to treat acute promyelocytic leukemia (APL), is found to cause QT interval prolongation during APL therapy ${ }^{[6]}$. Arsenic trioxide inhibits hERG channel expression without significant channel-blocking activity $^{[7]}$. In addition, we previously discovered that ceramide was not able to alter hERG current by acute application (superfusion for $25 \mathrm{~min}$ ) but rather caused pronounced hERG current inhibition after prolonged incubation for $10 \mathrm{~h}^{[8]}$. An increas- 
ing number of studies are shifting their focus from channel blockade to other aspects of channel regulation. Toxicological mechanisms are often found to function within the biochemical and molecular aspects of physiological processes. Indeed, recent years have witnessed the emergence of a deeper understanding of the biochemical and molecular factors underlying hERG regulation and the corresponding rescue strategies for hERG dysfunction.

hERG channel biogenesis is a multifaceted process. The hERG gene $(\mathrm{KCNH} 2)$ is transcribed into mRNA in the nucleus. It is believed that only the mRNA that is successfully processed through the nonsense-mediated mRNA decay (NMD) checkpoint can be translated in the endoplasmic reticulum $(E R)^{[9]}$. Nascent hERG channels are formed in the ER after proper protein folding, subunit assembly, phosphorylation, and initial glycosylation. Through the ER-Golgi interaction complex (ERGIC), partially glycosylated hERG enters the Golgi apparatus and undergoes complete glycosylation. Finally, the channel is sorted, carried and inserted into the plasma membrane ${ }^{[10]}$. Meanwhile, channels already present on the plasma membrane are dynamically internalized and recycled.

Almost every aspect of hERG channel biogenesis could be potentially affected by genetic or pharmacological factors in a manner that contributes to LQTS. For example, approximately $30 \%$ of heterozygous gene mutations of the $\mathrm{KCNH} 2$ gene are premature stop codons and subject to NMD, which leads to a $50 \%$ functional loss of the hERG channel ${ }^{[1]}$. Point mutations G601S and T473P result in hERG trafficking deficiency, and T473P even induces severe TdP and cardiac arrest ${ }^{[11,12]}$. hERG protein folding can be disturbed by drugs like arsenic trioxide $^{[7]}$. hERG maturation and exportation from the ER are arrested by pentamidine, an antiprotozoal drug ${ }^{[13]}$. Moreover, degradation of the hERG protein can be increased by ceramide $^{[14]}$.

Given the close relationship between hERG channel dysfunction and LQTS, rescue strategies for hERG channel dysfunction are being developed. For example, low temperature $\left(27^{\circ} \mathrm{C}\right)$ is commonly applied as a rescue strategy by stabilizing the conformation of the hERG channel ${ }^{[15,16]}$. In addition, the hERG channel blocker E-4031 is able to remove the traffickingdeficient G601S-hERG channel from a microtubule-dependent quality control compartment and increase export from the $\mathrm{ER}^{[17]}$. Meanwhile, the hERG channel activator RPR260243 can slow down hERG channel deactivation, increase $\mathrm{I}_{[\mathrm{Kr}]}$ and reverse dofentilide-induced action potential duration (APD) and prolongation ${ }^{[18-20]}$. Rescue strategies are also being developed from the biological regulating factors of the hERG channel. Overexpression of Hsp90 successfully rescues expression of the A422T-hERG mutant ${ }^{[21]}$.

This article provides an overview of known mechanisms underlying hERG channel regulation, with an emphasis on biochemical and molecular components of hERG biogenesis and possible rescue strategies for hERG dysfunction.

\section{Channel biogenesis and regulating network}

The biological regulation of hERG in the nucleus and ER is summarized in Figure 1.

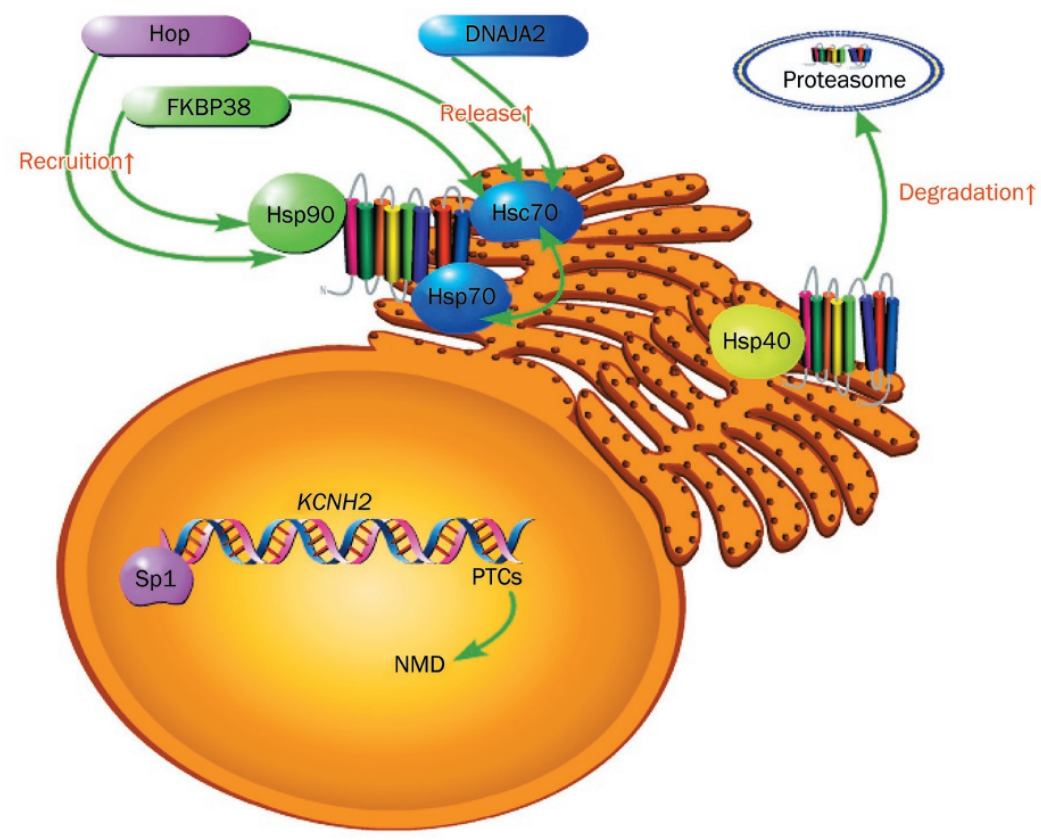

Figure 1. Biological regulations of hERG in nucleus and at ER. Transcription of the hERG channel (KCNH2 gene) is under the regulation of transcription factor Sp1 and the NMD mechanism. Hsc70 and Hsp70 at ER are responsible for early folding of hERG while Hsp90 in the cytoplasm is responsible for late folding. Hop, DNAJA2, and FKBP38 assist hERG release from Hsc70. Hop and FKBP38 aid hERG to recruit Hsp90 for its maturation. Hsp40 promotes degradation of $\mathrm{hERG}$ in the proteasome. 


\section{Transcription in nucleus}

Research on hERG channel transcription is limited and centers primarily on the NMD of premature mRNA. NMD participates mainly in gene expression regulation and mRNA quality control. It has been reported that $30 \%$ of $\mathrm{KCNH} 2$ gene mutations induce NMD, which is enough to cause a $50 \%$ reduction of hERG current thereby decreasing $I_{[\mathrm{Kr}]}^{[1]}$. It has also been reported that an extensively expressed transcriptional factor, SP1, up-regulates hERG mRNA. Furthermore, drugs may also rescue arsenic trioxide-induced hERG deficiency through upregulation of $S P 1^{[22]}$. Thus, NMD and SP1 might be good entry points through which interference of hERG dysfunction may occur.

\section{Translation and trafficking through ER and Golgi apparatus}

The initial hERG protein is translated in the ER. The initial, immature protein undergoes a series of complex modifications before being transported to the cell membrane. Generally speaking, native hERG protein is folded and assembled with the assistance of chaperones ${ }^{[1,23]}$. Properly folded and assembled hERG protein is coated in the COPII vesicle, transported forward to interact with ERGIC and arrives at the Golgi apparatus where it undergoes full-glycosylation ${ }^{[1,11]}$.

\section{Channel folding and assembling regulation at ER}

hERG channel folding and assembly are under the regulation of a complicated cyclic chaperone system. Among the numerous chaperones, Hsp/c70, Hsp90, Hop, FKBP38, Hsp40, DJA, 14-3-3 protein and GM130 have been best characterized.

Heat shock protein $70 \mathrm{kDa}(\mathrm{Hsp} 70)$ and its cognate (Hsc70) participate in the folding of native hERG while $90 \mathrm{kDa}$ heat shock protein (Hsp90) is involved in the maturation of latephase hERG ${ }^{[23,24]}$. It is believed that Hsp70 and Hsc70 bind with and regulate the hERG channel in a reciprocal way ${ }^{[25]}$. DNAJA2 and Hop assist in hERG release from Hsc70 ${ }^{[26,27]}$. Hop also aids the hERG protein in recruiting Hsp90 ${ }^{[27]}$. Hsp90 effectively inhibits mistakenly-folded protein aggregation and promotes hERG maturation ${ }^{[23]}$. FKBP38, a 38-kDa FK506binding protein, interacts both with Hsc70 and Hsp90 and may also be a prerequisite for hERG export from the $\mathrm{ER}^{[27]}$. Specifically, hERG degradation is thought to be regulated by Hsp40. DJA1 and DJA2, type-I of Hsp40, can decrease the interaction between hERG and Hsc70 thus promoting early degradation of $\mathrm{hERG}^{[28]}$. A more recent study demonstrated that the N-terminal Cap of the hERG channel is vital for the stability of the Per-Arnt-Sim (PAS) domain, hERG assembly and hERG trafficking ${ }^{[29]}$.

Importantly, drugs commonly interfere with channel folding and assembly, both of which are also targeted for rescue of channel dysfunction. It has been confirmed that arsenic trioxide inhibits the hERG channel by disturbing its interactions with Hsp90 and Hsp70 ${ }^{[7]}$. While trafficking deficiency of A422T-hERG can be corrected by Hsp90 overexpression ${ }^{[21]}$. FKBP38 has also been shown to partially restore trafficking of F805C-hERG ${ }^{[25]}$. Therefore, channel folding and assembly are most likely additional promising entry points for interfering with hERG dysfunction.

\section{Forward trafficking from ER to Golgi}

Properly folded and assembled hERG protein is coated in COPII and trafficked to the Golgi. It is believed that forward trafficking in the COPII vesicle is regulated by GTPase Sar1 while backward trafficking in the COPI vesicle is under the regulation of GTPase $A R F 1^{[30]}$. Rab11B is another regulating factor that inhibits forward trafficking of the hERG channel ${ }^{[30]}$. At the Golgi, GM130 is the commonly recognized chaperone that regulates hERG channel trafficking. GM130 participates in orienting the channel toward the Golgi by interacting with the $C$ terminal of the hERG channel. However, GM130 overexpression causes hERG current reduction. Furthermore, GM130 might act as a checkpoint for hERG maturation ${ }^{[31]}$.

It is well known that most studies on channel trafficking are focused on the ER. However, considering the importance of full glycosylation of the hERG channel at the Golgi, a deeper understanding of the characteristics of the pattern of regulation at the Golgi is essential. Moreover, hypoxia-induced decreased biogenesis of the hERG channel is reported to result from enhanced ROS formation at the mitochondria ${ }^{[32]}$. These observations suggest that the physiological and pathological factors taking place in the Golgi and mitochondria are potentially of great clinical significance.

\section{Quality control}

The trafficking process is under the regulation of a strictmonitoring mechanism called quality control (QC). QC occurs mainly in the ER. When the hERG protein is unfolded or misfolded, QC will activate the unfolded protein response [UPR, also called endoplasmic reticulum stress response (ERS)] to prevent abnormal forward trafficking. Specifically, chaperones are overexpressed to assist protein folding ${ }^{[17]}$. Mistakenly-folded hERG protein is fated to be marked with the C-terminal of the Hsp70-interacting protein (CHIP) and ubiquitin, and degraded at the proteasome by the endoplasmic reticulum-associated degradation (ERAD) mechanism ${ }^{[1]}$. For example, G572R-hERG and E637K-hERG mutants upregulate the activating transcription factor 6 (ATF6)-one early responder of UPR. Downstream targets of ATF6, calnexin and calreticulin, are further increased to help hERG channel folding $^{[17]}$.

QC is an essential mechanism that guarantees the correction and clearance of abnormal channels. Understanding this mechanism will help to clarify more physiological and pathological mechanisms and will aid in the discovery of more rescue approaches for hERG dysfunction. This is summarized in Figure 2.

\section{Endocytosis and recycling on cell membrane}

The hERG protein that successfully reaches the Golgi is then fully glycosylated. Afterwards, the channel is sorted, sublocated and finally transported to the cell membrane. There, the hERG channel is dynamically internalized into the cytoplasm and recycled back to the membrane ${ }^{[1,23]}$. 


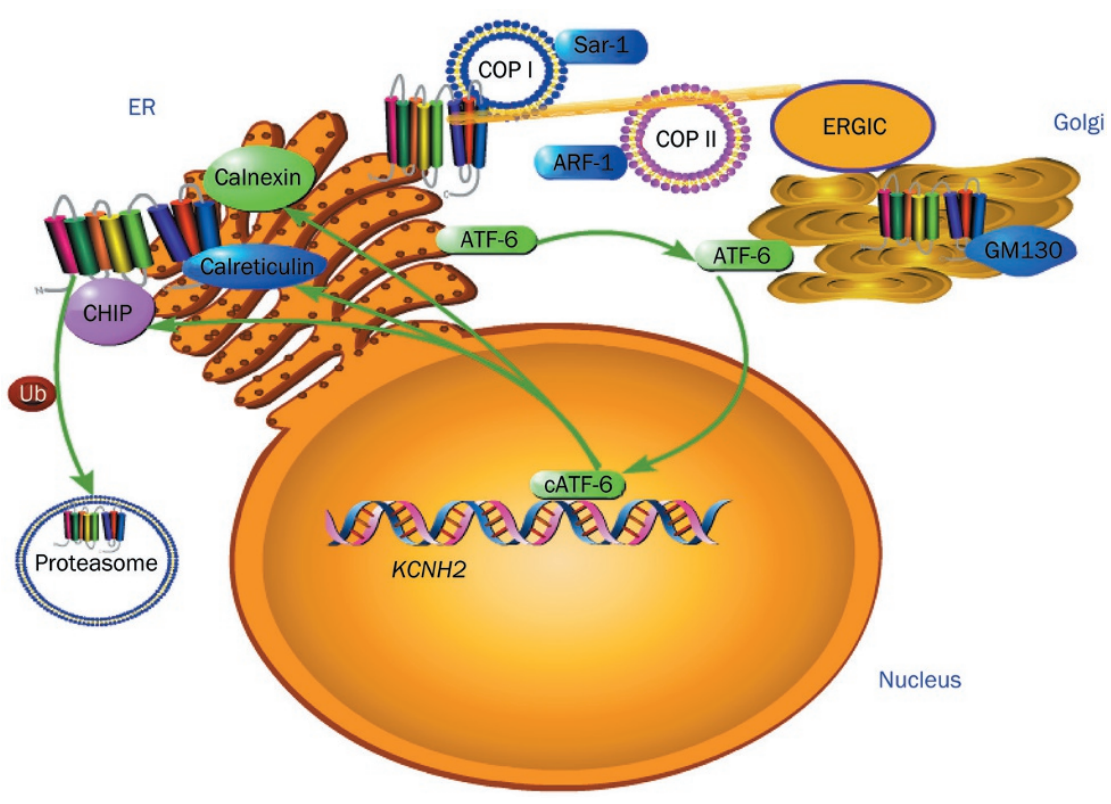

Figure 2. Quality control of hERG at ER. Normal hERG is coated in COPII (regulated by ARF-1) to forwardly traffick to the Golgi and interact with GM130. Abnormal hERG is coated in COPI (regulation by Sar-1) and backwardly trafficked to the ER; accumulation of abnormal hERG (especially unfolded hERG) will activate UPR; ATF- 6 will shuffle from the ER to the Golgi, ATF- 6 is cut to form cleaved-ATF- 6 and to travel into the nucleus and upregulate calnexin and calreticulin to assist hERG folding; CATF- 6 can, however, promote interaction between hERG and CHIP, then hERG is recognized and marked with ubiquitin to signal its degradation in the proteasome.

It is known that many transmembrane proteins lack cytoplasmic sequences that are required for recruitment and internalization into clathrin-coated vesicles ${ }^{[33]}$. Caveolin (Cav), dynamin and Arf6 are the most commonly reported members of clathrin-independent proteins that regulate protein internalization, including hERG channel internalization. Caveolin-3 (cav3) and caveolin-1 (cav1) have been reported to co-localize with hERG protein on the cell membrane and regulate channel internalization ${ }^{[14]}$. In addition, it has been shown that caveolar endocytosis, involving the caveolin, is dynamin-dependent ${ }^{[33]}$. One most recent study discovered that the membranal hERG protein undergoes rapid internalization in an Arf6-involving and dynamin-independent manner ${ }^{[34]}$. This process is summarized in Figure 3.

One fate of internalized hERG protein is to be ubiquitinated with the assistance of ubiquitin ligase and then degraded. For example, the WW domain of the ubiquitin ligase Nedd4-2 can interact with the PY motif of hERG channel ${ }^{[35]}$. Colocalization of Cav3, Nedd4-2 and hERG protein accelerates hERG ubiquitination and degradation by cav3 and Nedd42 , respectively ${ }^{[36,37]}$. Another fate of internalized hERG protein is to be recycled back to the membrane. Rab11 is one member that assists in this recycling process ${ }^{[37]}$. However, it has been reported that Rab4 overexpression reduces hERG channel expression by reducing degradation of Nedd4-2 $2^{[38]}$. Moreover, the serum and glucocorticoid-inducible kinase 3 (SGK3) and SGK1 have been shown to negatively regulate Nedd4-2-mediated ubiquitination and positively regulate Rab11-mediated recycling ${ }^{[37]}$. Thus, the membranal hERG protein appears to be under a dynamic balance of degradation and recycling.

Notably, probucol has been shown to speed up hERG degradation by influencing cav $1^{[39]}$. Ceramide increases hERG degradation by promoting ubiquitination ${ }^{[14]}$. Meanwhile, the trafficking deficiency of the cystic fibrosis transmembrane conductance regulator (F508-CFTR) is corrected by knocking down Nedd4-2. Knockdown of SGK1 completely blocked this rescue $^{[40]}$. Rab9 is able to rescue progesterone-induced hERG channel trafficking inhibition ${ }^{[30]}$. Therefore, Rab9, SGK, and Nedd4-2 are promising candidates that should be exploited for rescue of hERG channel dysfunction.

\section{Other regulation mechanisms}

It has been reported that prolonged APD and inhibited $I_{[\mathrm{Ks}]}$ can be normalized by an increase in $I_{[\mathrm{Kr}]}$, supporting the existence of complementary interactions between $I_{[\mathrm{Ks}]}$ and $I_{[\mathrm{Kr}]}^{[41]}$. $I_{[\mathrm{Kr}]}$ and $I_{[\mathrm{Ks}]}$ are both important currents that are responsible for cardiac repolarization. $I_{[\mathrm{Kr}]}$ is conducted by HERG (a subunit of $\left.I_{[\mathrm{Kr}]}\right)$ and KCNE2 ( $\beta$ subunit of $\left.I_{[\mathrm{Kr}]}\right)$ while $I_{[\mathrm{Ks}]}$ is conducted by $K C N E 1$ and $K C N Q 1^{[2]}$. It has been shown that the current of KCNE2-HERG co-expression bares a greater similarity to $I_{[\mathrm{Kr}]}$ than single HERG expression and that KCNE2 is also related to LQTS ${ }^{[42]}$. hERG can interact with KCNQ1-a via their $\mathrm{COOH}$ domains ${ }^{[12]}$ and trafficking-sufficient KCNQ1 can modulate the hERG channel ${ }^{[43]}$. Moreover, KCNE1 is reported to coimmunoprecipitate with hERG ${ }^{[4]}$ and KCNE2 may reduce hERG expression by accelerating degradation ${ }^{[45]}$. Thus, interactions among hERG, KCNQ1 and KCNE are essential for their expression ${ }^{[46]}$. Importantly, the polymorphisms in the hERG/MiRP1 $\mathrm{K}^{+}$-channel has been reported to potentially 


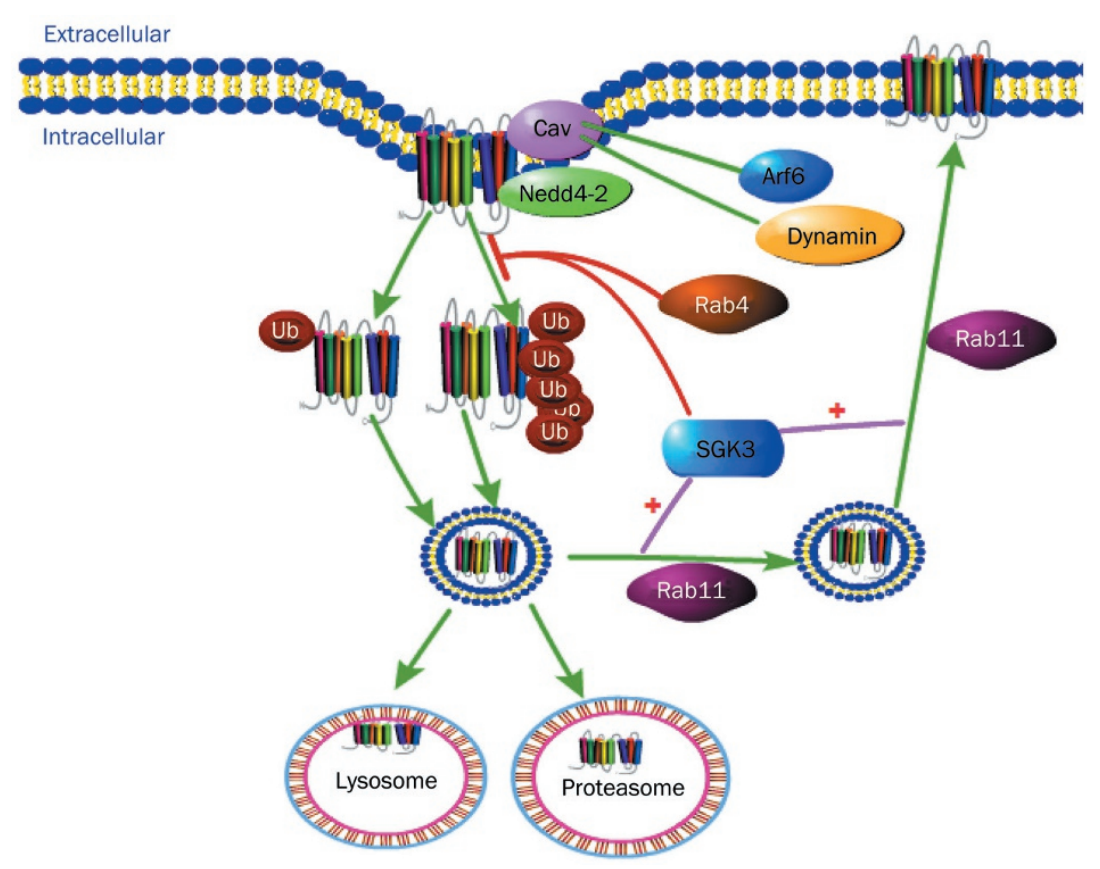

Figure 3. Endocytosis and recycling of hERG on cell membrane. Caveolin and Nedd4-2 both co-localize with hERG on the cell membrane. Instability of caveolin or Nedd4-2-dependent ubiquitination increases endocytosis of hERG. Caveolin-related endocytosis is Arf6-dependent and dynamin-dependent. Mono-ubiquitinized and multi-ubiquitinized hERG is degraded in the lysosome and proteasome, respectively. Rab11 promotes recyling of hERG back to cell membrane. Rab4 and SGK3 both decrease Nedd4-2-dependent ubiqtuination of hERG and SGK3 meanwhile promotes Rab11-dependent reclycing of hERG.

increase susceptibility to arrhythmia ${ }^{[47,48]}$. Clearly, future studies are needed to clarify the relationships among hERG, KCNQ1 and KCNE proteins and their intrinsic modulating mechanisms.

Ions, temperature, age, gender, and oxidative stress are other factors that regulate the hERG channel. For example, extracellular $\mathrm{K}^{+}$is a prerequisite for hERG channel function and membrane stability ${ }^{[46]}$. Low extracellular $\mathrm{K}^{+}$will induce channel mono-ubiquitination, and degradation in the lysosome ${ }^{[49]}$. Furthermore, internalization and degradation are accelerated under conditions of low extracellular $\mathrm{K}^{+[46,49]}$. In addition, it is believed that intracellular $\mathrm{K}^{+}$helps to stabilize $h E R G$ conformation thereby facilitating export from the $\mathrm{ER}^{[50]}$. hERG channel biogenesis rate and function are both decreased under conditions of anoxia due to the formation of ROS in the mitochondria ${ }^{[51]}$.

Notably, prolonged APD of R190Q-KCNQ1 can be normalized by hERG channel intervention and trafficking-sufficient KCNQ1 can also rescue decreased G601S-hERG/F805C-hERG channel expression ${ }^{[41,43]}$. Understanding these regulatory mechanisms may provide useful rescue strategies for hERG dysfunction.

\section{hERG channel and LQTS}

At present, the most common disease related to hERG channel dysfunction is LQTS. Patients with a prolonged QT interval are susceptible to arrhythmia including torsade de pointes and sudden cardiac death. The hERG abnormality associated with induction of LQTS includes acquired LQTS (aLQTS) that is mostly caused by drugs (as shown in Table 1) and congenital

Table 1. Mechanisms of hERG channel inhibition by representative drugs.

\begin{tabular}{lll}
\hline Mechanism types & Drug name & \multicolumn{1}{c}{ Detailed mechanisms } \\
\hline \multirow{2}{*}{ Direct block } & Dofetilide $^{54}$ & HERG current $\downarrow$; block at F656 \\
& Propafenrone $^{56}$ & HERG current $\downarrow$; structure with nitrogen, hydroxy, right angle \\
Trafficking defect & As $_{2} \mathrm{O}_{3}{ }^{7}$ & HERG current $\downarrow ; I_{\text {ca }}{ }^{2+-A P D-Q T \uparrow ; ~ t r a f f i c k i n g ~} \downarrow$, Hsp90-herg $\downarrow$ \\
& Pentamidine $^{13}$ & HERG current and expression $\downarrow$; ER retention \\
Degradation & Cisapride $^{58}$ & HERG current and expression $\downarrow$; ubiquitination-degradation \\
Multi-pathway & Probucol $^{23}$ & HERG current and expression $\downarrow ; I_{\mathrm{kr}}$-APD $\uparrow$; cav intererence \\
& Fluoxetine $^{62}$ & HERG current and expression $\downarrow$; block S6 area; traficking $\downarrow$ \\
& Desipramine $^{63}$ & HERG current and expression $\downarrow$; block; trafficking $\downarrow$; degrade $\uparrow$ \\
\hline
\end{tabular}




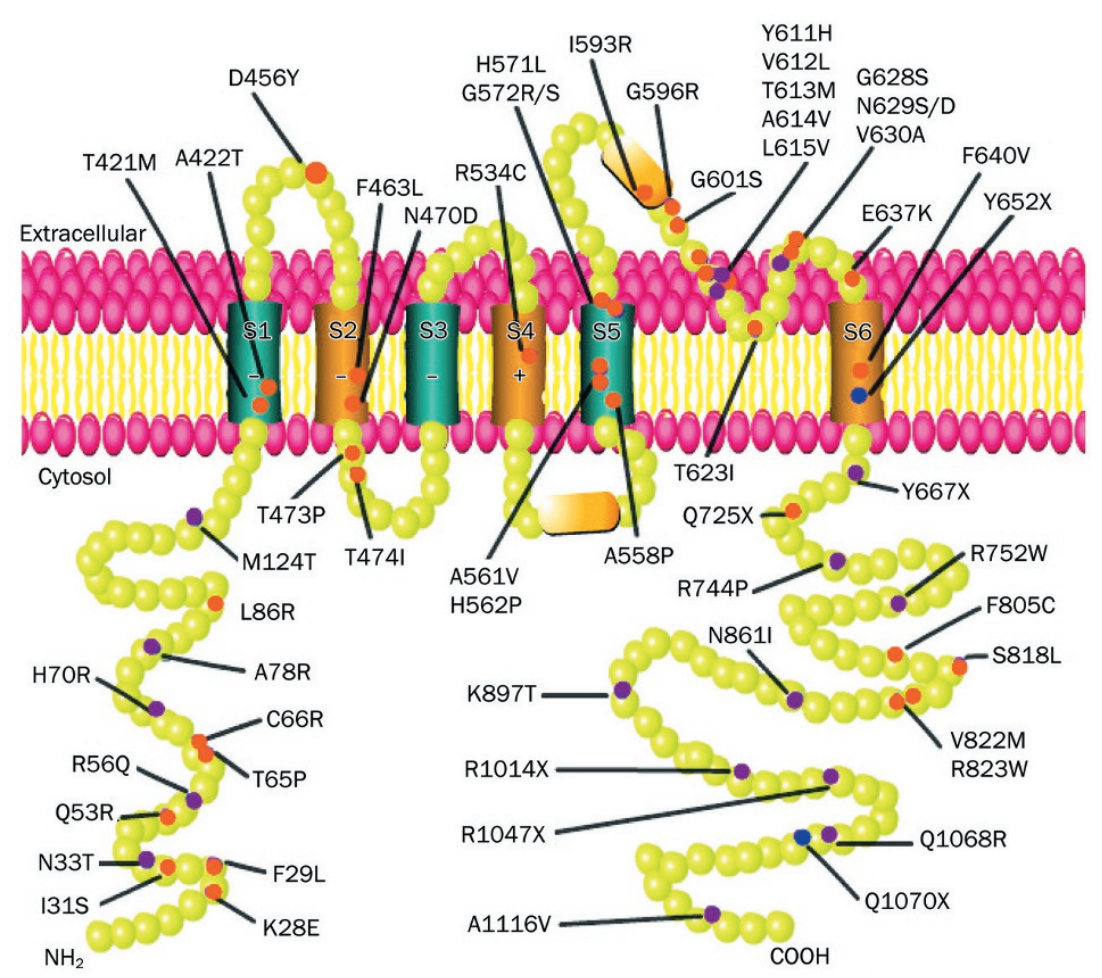

Figure 4. Schematics of LQT2 mutations. Each green ball represents an amino acid. Each purple, orange and blue point represents one mutation. In addition, the orange points represent mutations showing hERG channel trafficking deficiency and the blue points represent mutations being monitored by the NMD mechanism.

LQTS (cLQTS) that is caused by gene mutations (as shown in Figure 4).

\section{aLQTS of hERG channel}

Many factors may induce aLQTS including hypokalemia, anoxia and diverse drugs ${ }^{[49,51,52]}$. Drug-induced aLQTS is the most common type. Drugs like terfenadine have been removed from the market because of the adverse cardiac side effects caused by hERG channel inhibition. So far, approximately 136 drugs (www.crediblemeds.org; www.QTdrugs. org) have been reported to prolong the QT interval. It is surprising that such a diverse categories of drugs are capable of inhibiting the hERG channel including antiarrhythmic, antipsychotic and antidepressant drugs. For example, berberine inhibits the hERG channel causing APD prolongation ${ }^{[53]}$. Arsenic trioxide prolongs the QT interval by inhibiting hERG channel expression and increasing the calcium current $t^{[7]}$. The antidepressant drug, desipramine, induces QT prolongation through multiple pathways that affect the hERG channel. Therefore, the hERG channel is the most common cause for drug-induced aLQTS. These drugs impact the hERG channel mainly by direct blocking and by interfering with pathways involved in biogenesis.

\section{hERG channel blocking}

hERG channel blocking is the number one reason for druginduced hERG channel dysfunction. The hERG channel can be blocked at different sites by different drugs making it important to analyze the blocking process.

From the point of view of different binding sites, Phe656 and Thr652 are the two common sites for drug binding. For example, dofetilide binds to Phe656 to decrease hERG current ${ }^{[54]}$. While most drugs block the hERG channel through Phe656 and Tyr652, other sites are also targets. The blocking effect of E4031 and dofetilide on the hERG channel includes Val625, Ser624 and Thr623 (near the pore), Gly648 and Val659 (on the S6 domain), and S631A (between domain 5 and 6) ${ }^{[54,55]}$.

When considering different blocking compounds, compounds with nitrogen, hydroxy or a right angle can more easily inhibit the hERG channel ${ }^{[56]}$. In addition, an aromatic side chain on Tyr652 and a hydrophobic side chain on Phe656 are needed for cisapride-associated hERG current blocking ${ }^{[57]}$. As drugs prefer to form п-п stacking interactions with Tyr652 and Phe656, Tyr652 and/or Phe656 mutations could reduce drug-associated hERG inhibition ${ }^{[58]}$. The hydrophobicity and aromatic character at Tyr623, Tyr652 and Phe656 are thought to be the main reasons for blocking ${ }^{[59]}$. Van der Waals' force present on the channel surface is also necessary for blocking ${ }^{[59]}$.

In conclusion, Tyr652, Phe656, and Tyr623 are the most essential sites for blocking. Val625, Ser624, Ser631, Gly648, and Val659 are also common sites for blocking. There are many different opportunities for blocking such as protonized nitrogen forming hydrogen bonds with the oxygen of carbonyl at Tyr623, a half aromatic ring interacting with п-п stacking at 
Tyr652 and the hydrophobic part can forming a hydrophobic interaction with the benzene ring at Phe656. New drugs that off-target these binding sites might be one good solution.

\section{hERG channel biogenesis interference}

Biogenesis disturbance, especially trafficking inhibition, is an emerging reason for drug-induced hERG dysfunction. Arsenic trioxide disturbs the hERG-chaperone interaction so as to impede channel trafficking ${ }^{[7]}$. Higher levels of miR-133 and lower levels of hERG expression are also associated with arsenic trioxide-induced QT interval prolongation ${ }^{[60]}$. Pentamidine binds with the hERG channel to arrest its maturation and inhibits exportation from the $\mathrm{ER}^{[13]}$. It has been reported that pentamidine with a phenyl ring or substituents that can attach to the hERG channel has the largest inhibitory effect on the hERG channel ${ }^{[61]}$.

Drugs also influence the hERG channel by their impact on degradation. Probucol speeds up hERG channel degradation through its effects on low-density lipoprotein (LDL) and $\operatorname{cav} 1^{[23]}$. Ceramide increases channel degradation at the lysosome by promoting channel ubiquitination ${ }^{[14]}$.

In fact, many drugs interfere with the hERG channel through multiple pathways. Fluoxetine both blocks hERG current and reduces channel trafficking ${ }^{[62]}$. Even desipramine inhibits the hERG channel by blocking, trafficking inhibition, promoting internalization and accelerating ubiquitinationdegradation ${ }^{[63]}$.

As the majority of the processes involved in biogenesis of the hERG channel are affected by diverse drugs, rescue strategies of hERG dysfunction should be specific to their targets. For example, drug-induced trafficking defects can be minimized if certain chemical features are avoided or removed ${ }^{[61]}$.

\section{cLQTS of hERG channel}

hERG channel mutation-induced cLQT2 account for approximately $45 \%$ of identified LQTS ${ }^{[64]}$. It was reported in 2009 that $62 \%$ of $\mathrm{KCNH} 2$ mutations were missense mutations, $24 \%$ were frame-shift mutations, and $14 \%$ were nonsense and deletion mutations ${ }^{[65]}$. Up to $90 \%$ of missense mutations affect channel function by trafficking inhibition ${ }^{[6]]}$. Mutations are located throughout the entire channel: intracellular domains (52\%), trans-membrane domains $(30 \%)$, pore areas $(12 \%)$, and extracellular segments $(6 \%)^{[64]}$.

\section{Common mutation characteristics}

As shown in Figure 4, mutations are located throughout the hERG channel. Mutations in different areas have different impacts on the hERG channel. In the pore area, G601S manifests as inhibited trafficking while $\mathrm{Y} 611 \mathrm{H}$ manifests as increased ubiquitination and degradation ${ }^{[11,67]}$. The transmembrane mutation T473P causes severe TdP and cardiac arrest $^{[12]}$. Mutations around T473P such as D456Y, F463 L, N470D, and T474I all manifest as trafficking inhibition with dominant negative effect ${ }^{[12]}$. Therefore, it has been inferred that mutations between S2 and the S2-S3 linker probably have serious manifestations. Mutations at the PAS domain do not frequently inhibit trafficking. It has been shown that K28E, F29L, G53R, C66G, L86R cause trafficking deficiency while N33T, R56Q, H70R, A78R do not ${ }^{[68]}$. N33T and R56Q are likely to directly influence the membranal hERG channel, while H70R and A78R probably indirectly affect the hERG channel by interacting with surrounding hydrophobic areas ${ }^{[69]}$. The red points in Figure 4 show the locations of traffickingdeficient mutants.

In summary, mutations at the pore and trans-membrane domains probably disturb channel trafficking and have severe consequences, while mutations at the PAS domain and the C terminal are likely to have milder consequences. Rescue of the hERG channel dysfunction should consider these types of characteristics and avoid such mutations.

\section{Other mutation characteristics}

Considering hERG channel dysfunction mechanism, some mutation-associated hERG inhibitions are regulated by QC. For example, ATF-6, Grp78, Grp94, and calreticulin are all activated to assist in I593R-hERG folding ${ }^{[68]}$. UPR is also activated by the E637K-hERG and G572R-hERG mutations ${ }^{[17]}$. In addition, some mutations can alter the kinetics of hERG channel. R744P-hERG causes hERG channel dysfunction by reducing activation ${ }^{[70]}$. T421M-hERG manifests as both trafficking inhibition and changes in kinetics ${ }^{[71]}$. Furthermore, mRNA is decreased via the NMD mechanism for both Q1070X-hERG and Y652X-hERG ${ }^{[72,73]}$. Q725X-hERG and R1014X-hERG also cause a $\mathrm{C}$ terminal premature truncation ${ }^{[7]}$. Recently, a study defined the required segments for susceptibility to NMDassociated LQTS ${ }^{[7]}$.

Regarding the type of mutation, those that exclude nonsense mutations also play a certain role in cLQTS. Frame-shift mutations such as P1086fs $+32 X^{[76]}$, splicing mutations such as $2592+1 G>A^{[77]}$, and gene polymorphisms such as K897T, R1047L and Q1068R are all reported to induce cLQTS ${ }^{[76-78]}$. Specifically, patients carrying the A1116V-hERG mutation without K897T gene polymorphism manifest normal hERG function, while patients carrying A1116V-hERG mutation and K897T gene polymorphism manifest hERG current inhibition $^{[79]}$

Therefore, mechanisms that exclude trafficking inhibition and mutations that exclude nonsense mutations cannot be neglected. Gene scanning should be broad enough to screen out dangerous factors, especially those mentioned above.

\section{Correlation between aLQTS and cLQTS}

Research investigating the correlations between aLQTS and cLQTS is limited. One study discovered DNA variants in a patient who experienced drug-induced $\mathrm{TdP}^{[80]}$. Probucol is also found to accelerate M124T-hERG-associated current inhibition $^{[81]}$. Furthermore, hERG gene polymorphism is also correlated with drug-induced hERG channel abnormality ${ }^{[39,82]}$.

As patients who carry risky gene mutations are more susceptible to LQTS when they are treated with potentially dangerous drugs, it is necessary to further clarify the correlations between aLQTS and cLQTS. When both aLQTS and cLQTS 
are taken into consideration, cross-link rescue strategy seems to hold great promise.

\section{Existing and potential rescue strategies}

At present, rescue strategies for hERG channel dysfunction mainly include channel blockers, activators, biogenesis regulating factors and other methods. All rescue strategies are summarized in Table 2. Analysis of the merits and demerits of these strategies will help to develop the most appropriate rescue strategies that can be clinically applied.

\section{hERG channel activators}

Activators seem to be the most promising strategy for rescue hERG channel dysfunction as well as LQTS in vivo. So far, there are approximately 13 reported hERG activators: RPR260243, PD118057, PD307243, NS1643, NS3623, ICA105574, KB130015, A-935142, mallotoxin, matrine, oxymatrine, carbachol, and N-[N-(N-acetyl-L-leucyl)-L-leucyl]-L-norleucine $(\mathrm{ALLN})^{[18,83-87]}$.

Activators prefer to regulate the hERG channel by altering channel kinetics ${ }^{[18]}$. Activators are divided into seven types based on their mechanism of activation: (1) RPR260243 mainly affects the hERG channel by altering deactivation $^{[18,88]}$. (2) PD118057, PD307243, NS1643, NS3623, and ICA-105574 all influence the hERG channel by regulating

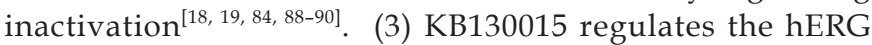
channel by increasing activation ${ }^{[18]}$. (4) A-935142 and mallotoxin both regulate the hERG channel by changing multiple kinetics ${ }^{[20,83]}$. (5) Matrine and oxymatrine influence the hERG channel by up-regulating transcription instead of changing kinetics $^{[22,85]}$. (6) Carbachol increases Nedd4-2 phosphorylation, slows down channel degradation and thus increases hERG channel expression ${ }^{[86]}$. (7) ALLN, a known calpain1/2 and proteasome inhibitor, rescues the LQTS2 phenotype by restoring trafficking ${ }^{[87]}$.

Importantly, many activators are capable of shortening pro- longed APD and the QT interval. For example, RPR260243 is able to reverse dofentilide-induced APD prolongation by slowing down $\mathrm{hERG}$ deactivation to increase $I_{[\mathrm{kr}]}^{[18-20]}$. In addition, PD118057 can reduce hERG inactivation, shift up inactivation potential and prevent dofetilide-caused QT interval prolongation $^{[18,84]}$. However, PD118057 fails to correct the dominant-negative effect of the E637K-hERG ${ }^{[91]}$. ICA-105574 can also disable hERG inactivation and shorten the QT interval in cardiomyocytes ${ }^{[18,84]}$. Moreover, matrine and oxymatrine can also rescue arsenic trioxide-induced hERG deficiency and APD prolongation ${ }^{[22]}$. ALLN successfully reduces spontaneous arrhythmogenic episodes and markedly reduces $\mathrm{APD}_{70 / 90}$ of LQTS-specific cardiomyocytes derived from human-induced pluripotent stem cells ${ }^{[87]}$.

In conclusion, the hERG channel activators are promising agents to use in the rescue of drug-induced LQTS in vivo. Activators such as RPR260243, PD118075, ICA-105574, and ALLN show the most potential. Matrine and oxymatrine are also promising for the rescue of other types of hERG dysfunction owing to their role in increasing transcription.

\section{hERG channel blockers}

hERG channel blockers are widely used to rescue hERG dysfunction in vitro. E-4031, astemizole, fexofenadine and dofetilide are most often chosen. For example, E-4031 is able to remove trafficking-deficient G601S-hERG from the microtubule-dependent QC compartment and increase ER export of the mutant hERG channel ${ }^{[17]}$. In addition, astemizole and fexofenadine are both reported to rescue expression deficiency of N470D-hERG ${ }^{[15,92]}$. Dofetilide is reported to correct pentamidine-induced hERG defects ${ }^{[61]}$.

Nevertheless, there are quite a few barriers due to blockers that limit their application. First, as blockers bind to the intra-cavity of the hERG channel, the rescued channel might be non-conducting ${ }^{[1]}$. For example, under conditions of low intracellular potassium, the astemizole-rescued channel is

Table 2. Representative rescue strategies.

\begin{tabular}{lll}
\hline Rescue strategy & Name & Mechanism \\
\hline \multirow{2}{*}{ Activators } & RPR260243 & Deactivation $\downarrow$ \\
& P118057, PD307243, NS1643, NS3623, ICA-105574 & Inactivation $\downarrow$ \\
& KB-130015 & Activation $\uparrow$ \\
& A-935142, mallotoxin & Multi-kinetics influence \\
& Matrine, oxymatrine & Transcription $\uparrow$ \\
& Carbachol & Degradation $\downarrow$ \\
Blockers & ALLN & Re-trafficking $\uparrow$ \\
& Fexofenadine & Competive binding \\
Promising strategies & E-4031, astemizole & Trafficking $\uparrow$ or recycling \\
& FKBP38, Hsp90, Rab9 & Specific-targeting interference \\
& AMO, siRNA, iPSC & Mutual interaction \\
KCNQ1 & Glycosylation modification \\
& KM57, KM60 & Conformatinstabalization \\
\hline
\end{tabular}


non-conducting ${ }^{[50]}$. Second, rescue concentrations of blockers are usually higher than their blocking concentrations, which restricts their application ${ }^{[92]}$. However, in the case of fexofenadine, the rescue concentration is 350 times lower than its blocking concentration on N470D-hERG ${ }^{[92]}$. Therefore, drugs that are capable of rescuing hERG dysfunction and show a low affinity for the hERG channel are promising. Third, the rescue effects of blockers are often related to their binding sites within the hERG channel and thus, many types of LQTS are not sensitive. Hypoxia-induced hERG trafficking deficiency or T473P-hERG-associated severe TdP can not be rescued by blockers ${ }^{[14]}$. Fexofenadine is also unable to rescue V822MhERG function ${ }^{[92]}$.

As a result, hERG channel blockers may not be the most promising in vivo strategy. However, fexofenadine's lower rescuing than blocking concentration makes it a potential rescue of hERG dysfunction clinically.

\section{Potential rescue strategies targeting hERG channel}

Not surprisingly, there is great potential to develop rescue strategies from factors that regulate the hERG channel biogenesis. Regulating factors such as FKBP38, Rab9/11B, Hsp90, SGK, Nedd4-2, and KCNQ are the most promising. FKBP38 promotes hERG trafficking and is able to rescue trafficking deficiency of F805C-hERG ${ }^{[27]}$. Rab9 is also able to rescue progesterone-induced hERG trafficking inhibition ${ }^{[30]}$. Furthermore, expression of trafficking-deficient A422T-hERG can be rescued by overexpressing Hsp90 ${ }^{[21]}$. Finally, traffickingsufficient KCNQ1 can restore G601S/F805C-hERG channel expression $^{[41,43]}$.

The rescuing effects of the previously mentioned strategies lack specificity. SiRNA interference and the AMO strategy, which targets specific objectives, are designed to rescue the hERG channel defects. For instance, E637K-hERG-targeting siRNA reduces the expression of the mutant channel without affecting expression of the wild type channel ${ }^{[93]}$. Allele-specific RNA interference, which targets c.G1681A-KCNH2 mRNA, successfully normalizes hERG current and APD ${ }^{[94]}$. Moreover, pluripotent stem cells (iPSCs) bring great hope for rescuing of hERG dysfunction ${ }^{[95]}$.

Although most of these studies are in vitro, because of their specificity, regulating factors and gene-targeting strategies are quite promising for clinical application.

\section{Other rescuing strategies}

Low temperature, glycerol, DMSO and TMO are commonly used to rescue hERG dysfunction ${ }^{[15,16,23,69]}$. Temperature $27^{\circ} \mathrm{C}$ is often chosen for low-temperature incubation ${ }^{[15,16]}$. hERG channel mutations such as K28E, F29L, D456Y, N470D, G601S, F805C, and V822M and hypoxia-induced hERG trafficking inhibition all can be rescued by low-temperature incubation $^{[23,69]}$. Chemical chaperones such as glycerol, DMSO and TMO are believed to stabilize hERG channel conformation ${ }^{[23]}$.

Interestingly, chemicals that can rescue other abnormalities in other channels are capable of rescuing hERG dysfunction as well. KM57 and KM60, chemicals that can rescue expres- sion deficiency of the CFTR channel, are also able to rescue the G601S-hERG channel deficiency. Specifically, KM57 and KM60 might play their rescuing role by modifying glycosylation of the hERG channel ${ }^{[96]}$. This is especially useful when more than one type of channel mutation occurs. In addition, we previously found that acidification markedly potentiated dofetilide-induced blockade of the hERG channel while it weakened quiniding/azimilide-induced hERG inhibition ${ }^{[97]}$. Consideration of blood $\mathrm{pH}$ should help to reduce druginduced hERG channel dysfunction.

\section{Conclusion and prospect}

In summary, hERG channel biogenesis and the regulating network are quite complex, especially in regard to functions of the ER and dynamic cycling on the cell membrane. There are many biochemical and molecular targets that may induce channel disorders including LQTS. In addition, LQTSassociated mutations spread throughout the whole area of the hERG channel, particularly in the pore area and trans-membrane segments. Trafficking is most often affected. Rescue strategies include activators, blockers, biogenesis regulating factors and other methods.

Looking toward the future, comprehensive consideration and individual treatment of the hERG channel disorder and LQTS are the trends. Activators seem to be the most promising rescue strategy for hERG channel dysfunction and LQTS. Importantly, rescue strategies with great potential await exploitation in the biogenesis regulation network, especially the trafficking process.

\section{Acknowledgements}

This work was supported by grants from the National Natural Science Foundation of China (№ 31173050 and 30973530) and the Key Program of the National Natural Science Foundation of China (№ 81230081).

\section{References}

1 Vandenberg JI, Perry MD, Perrin MJ, Mann SA, Ke Y, Hill AP. hERG K ${ }^{+}$ channels: structure, function, and clinical significance. Physiol Rev 2012; 92: 1393-478.

2 Sanguinetii MC, Tristani-Firouzi M. hERG potassium channels and cardiac arrhythmia. Nature 2006; 440: 463-9.

3 Babcock JJ, Li M. hERG channel function: beyond long QT. Acta Pharmacol Sin 2013; 34: 329-35.

4 Warmke JW, Ganetzky B. A family of potassium channels genes related to eag in Drosophila and mamals. Proc Natl Acad Sci U S A 1994; 91: 3438-43.

5 Sanguinetti MC, Jiang CG, Curran ME, Keating MT. A mechanistic link between an inherited and an acquired cardiac arrhythmia: HERG encodes the Ikr potassium channel. Cell 1995; 81: 299-307.

6 Zhou J, Meng R, Li XX, Lv CF, Fan SJ, Yang BF. The effect of arsenic trioxide on QT interval prolongation during APL therapy. Chin Med J 2003; 116: 1764-6.

7 Ficker E, Kuryshev YA, Dennis AT, Obejero-Paz C, Wang L, Hawryluk $P$, et al. Mechanisms of arsenic-induced prolongation of cardiac repolarization. Mol Pharmacol 2004; 66: 33-44.

8 Bai YL, Wang JX, Shan HL, Lv YJ, Zhang Y, Luo XB, et al. Sphingolipid metabolite ceramide causes metabolic perturbation contributing to 
HERG K ${ }^{+}$channel dysfuncion. Cell Physiol Biochem 2007; 20: 42940.

9 Gong Q, Zhang L, Vincent GM, Horne BD, Zhou Z. Nonsense mutations in hERG cause a decrease in mutant mRNA transcripts by nonsense-mediated mRNA decay in human long-QT syndrome. Circulation 2007; 116: 17-24.

10 Hebert DN, Molinari M. In and out of the ER: protein folding, quality control, degradation, and related human diseases. Physiol Rev 2007; 87: $1377-408$.

11 Smith JL, McBride CM, Nataraj PS, Bartos DC, January CT, Delisle BP. Trafficking-deficient hERG $\mathrm{K}^{+}$channels linked to long QT syndrome are regulated by a microtubule-dependent quality control compartment in the ER. Am J Physiol Cell Physiol 2011; 301: C75-85.

12 Liu L, Hayashi K, Kaneda T, Ino H, Fujino N, Uchiyama K, et al. A novel mutation in the transmembrane non-pore region of the $\mathrm{KCNH} 2$ gene causes severe clinical manifestations of long QT syndrome. Heart Rhythm 2013; 10: 61-7.

13 Dennis AT, Wang L, Wan H, Nassal D, Deschenes I, Ficker E. Molecular determinants of pentamidine-induced hERG trafficking inhibition. Mol Pharmacol 2012; 81: 198-209.

14 Chapman H, Ramstrom C, Korhonen L, Laine M, Wann KT, Lindholm D, et al. Downregulation of the HERG $(\mathrm{KCNH} 2) \mathrm{K}^{+}$channel by ceramide: evidence for ubiquitin-mediated lysosomal degradation. J Cell Sci 2005; 118: 5325-34.

15 Zhou Z, Gong Q, January CT. Correction of defective protein trafficking of a mutant HERG potassium channel in human long QT syndrome pharmacological and temperature effects. J Bio Chem 1999; 274 : 31123-6.

16 Chen MX, Sandow SL, Doceul V, Chen YH, Harper H, Hamiton B, et al. Improved functional expression of recombinant human ether-ago-go (hERG) $\mathrm{K}$ channels by cultivation at reduced temperature. BMC Biotechnol 2007; 7: 93.

17 Wang Y, Huang X, Zhou J, Yang X, Li D, Mao H, et al. Traffickingdeficient G572R-hERG and E637K-hERG activate stress and clearance pathways in endoplasmic reticulum. PLoS One 2012; 7: e29885.

18 Perry M, Sanguinetti M, Mitcheson J. Revealing the structural basis of action of hERG potassium channel activators and blockers. J Physiol 2010; 588: 3157-67.

19 Kang J, Chen XL, Wang H, Ji J, Cheng H, Incardona J, et al. Discovery of a small molecule activator of the human ether-a-go-go-related gene (HERG) cardiac K channel. Mol Pharmacol 2005; 67: 827-36.

20 Liu X, Limberis JT, Su Z, Houseman K, Diaz GJ, Gintant GA, et al. Characterization of A-935142, a hERG enhancer, in the presence and absence of standard hERG blockers. Life Sci 2012; 90: 607-11.

21 Guo J, Zhang X, Hu Z, Zhuang Z, Zhu Z, Chen Z, et al. A422T mutation in HERG potassium channel retained in ER is rescurable by pharmacologic or molecular chaperones. Biochem Biophys Res Commun 2012; 422: 305-10.

22 Zhang Y, Dong ZX, Jin L, Zhang K, Zhao X, Fu J, et al. Arsenic trioxideinduce $h E R G K$ channel deficiency can be rescued by matrine and oxymatrine through up-regulating transcription factor Sp1 expression. Biochem Pharmacol 2013; 85: 59-68.

23 Delisle BP, Anson BD, Rajamani S, January CT. Biology of cardiac arrhythmias ion channel protein trafficking. Circ Res 2004; 94 : 1418-28.

24 Ficker E, Dennis AT, Wang L, Brown AM. Role of the cytosolic chaperones Hsp70 and $\mathrm{Hsp9O}$ in maturation of the cardiac potassium channel HERG. Circ Res 2003; 92: e87-100.

25 Li P, Ninomiya H, Kurata Y, Kato M, Miake J, Yamamoto Y, et al. Reciprocal control of hERG stability by $\mathrm{Hsp} 70$ and $\mathrm{Hsc70}$ with implication for restoration of LQT2 mutant stability. Circ Res 2011; 108 :
458-68.

26 Baaklini I, Wong MJ, Hantouche C, Patel Y, Shrier A, Young JC. The DNAJA2 substrate release mechanism is essential for chaperonemediated folding. J Biol Chem 2012; 287: 41939-54.

27 Walker VE, Atanasiu R, Lam H, Shrier A. Co-chaperone FKBP38 promotes HERG trafficking. J Biol Chem 2007; 282: 23509-16.

28 Walker VE, Wong MJ, Atanasiu R, Hantouche C, Young JC, Shrier A. Hsp40 chaperones promote degradation of the HERG potassium channel. J Biol Chem 2010; 285: 3319-29.

29 Ke Y, Hunter MJ, Ng CA, Perry MD, Vandenberg Jl. Role of the cytoplasmic N-terminal cap and Per-Arnt-Sim (PAS) doman in trafficking and stabilization of Kv11.1 channels. J Biol Chem 2014; 289: 13782-91.

30 Delisle BP, Underkofler HA, Moungey BM, Slind JK, Kilby JA, Best $\mathrm{JM}$, et al. Small GTPase determinants for the Golgi processing and plasmalemmal expression of human ether-a-go-go related (hERG) $\mathrm{K}^{+}$ channels. J Biol Chem 2009; 284: 2844-53.

31 Roti EC, Myers CD, Ayers RA, Boatman DE, Delfosse SA, Chan EK, et al. Interaction with GM130 during HERG ion channel trafficking disruption by type 2 congenital long QT syndrome mutation. Human ether-a-go-go-related gene. J Biol Chem 2002; 277: 47779-85.

32 Nanduri J, Wang N, Bergson P, Yuan G, Ficker E, Prabhakar NR. Mitochondrial reactive oxygen species mediate hypoxic down-regulation of hERG channel protein. Biochem Biophys Res Commun 2008; 373 : 309-14.

33 Grant BD, Donaldson JG. Pathways and mechanisms of endocytosis recycling. Nat Rev Mol Cell Biol 2009; 10: 597-608.

34 Karnik R, Ludlow MJ, Abuarab N, Smith AJ, Hardy ME, Elliott DJ, et al. Endocytosis of hERG is clathrin-independent and involves Arf-6. PLoS One 2013; 8: e85630.

35 Albesa M, Grilo LS, Gavillet B, Abriel H. Nedd4-2-dependent ubiquitylation and regulation of the cardiac potassium channel hERG1. J Mol Cell Cardiol 2001; 51: 90-8.

36 Guo J, Wang T, Li X, Shallow H, Yang T, Li W, et al. Cell surface expression of human ether-a-go-go-related gene (hERG) channels is regulated by caveolin-3 protein via the ubiquitin ligase Nedd4-2. J Biol Chem 2012; 287: 33132-41.

37 Lamothe SM, Zhang S. The serum-and glucocorticoid-inducible kinase SGK1 and SGK3 regulate hERG channel expression via ubiquitin ligase Nedd4-2 and GTPase Rab11. J Biol Chem 2013; 288 : 15075-84.

38 Cui Z, Zhang S. Regulation of the human ether-a-go-go-related gene (hERG) channel by Rab4 through neural precursor cell-expressed developmentally downregulated protein 4-2 (Nedd4-2). J Biol Chem 2013; 288: 21876-86.

39 Guo J, Li X, Shallow H, Xu J, Yang T, Massaeli H, et al. Involvement of caveolin in probucol-induced reduction in hERG plasma-membrane expression. Mol Pharmacol 2011; 79: 806-13.

40 Caohuy H, Jozwik C, Pollard HB. Rescue of F508-CFTR by the SGK1/ Nedd4-2 signaling pathway. J Biol Chem 2009; 284: 25241-53.

41 Zhang H, Zou B, Yu H, Moretti A, Wang X, Yan W, et al. Modulation of hERG potassium channel gating normalizes action potential duration prolonged by dysfunctional KCNQ1 potassium channel. Proc Natl Acad Sci U S A 2012; 109: 11866-71.

42 Abbott GW, Sesti F, Splawski I, Buck ME, Lehmann MH, Timothy KW, et al. MiRP1 forms potassium channels with HERG and is associated with cardiac arrhythmia. Cell 1999; 97: 175-87.

43 Hayashi K, Shuai W, Sakamoto Y, Higashida H, Yamagishi M, Kupershmidt S. Traffcking-competent KCNQ1 variably influences the function of HERG long QT alleles. Heart Rhythm 2010; 7: 973-80.

44 Organ-Darling LE, Vernon AN, Giovanniello JR, Lu Y, Moshal K, Roder K, 
et al. Interaction betwee hERG and KCNQ1 $\alpha$-subunits are mediated by their C-termini and modulated by cAMP. Am J Physiol Heart Circ Physiol 2013; 304: H589-99.

45 Zhang M, Wang Y, Jiang M, Zankov DP, Chowdhury S, Kasirajan V, et al. KCNE2 protein is more abundant in ventricles than in atria and can accelerate hERG protein degradation in a phosphorylationdependent manner. Am J Physiol Heart Circ Physiol 2012; 302: H910-22.

46 Massaeli $\mathrm{H}$, Guo J, Xu J, Zhang S. Extracellular $\mathrm{K}^{+}$is a prerequisite for the function and plasma membrane stability of HERG channels. Circ Res 2010; 106: 1072-82.

47 Larsen LA, Andersen PS, Kanters J, Svendsen IH, Jacobsen JR, Vuust $J$, et al. Screening for mutations and polymorphisms in the genes KCNH2 and KCNE2 encoding the cardiac HERG/MiRP1 ion channel: implications for acquired and congenital long QT syndrome. Clin Chem 2001; 47: 1390-5.

48 Weerapura M, Nattel S, Chartier D, Caballero R, Hebert TE. A comparison of currents carried by HERG, with and without coexpression of MiRP1, and the native rapid delayed rectifier current-ls MiRP1 the missing link? J Physiol 2002; 540: 15-27.

49 Sun T, Guo J, Shallow H, Yang T, Xu J, Li W, et al. The role of monoubiquitination in endocytic degradation of human ether-a-go-go-related gene (hERG) channels under low $\mathrm{K}^{+}$conditions. J Biol Chem 2011; 286: 6751-9.

50 Wang L, Dennis AT, Trieu P, Charron F, Ethier N, Hebert TE, et al. Intracellular potassium stabilizes human ether-a-go-go-related gene channels for export from endoplasmic reticulum. Mol Pharmacol 2009; 75: 927-37.

51 Nanduri J, Bergson P, Wang N, Ficker E, prabhakar NR. Hypoxia inhibits maturation and trafficking of $\mathrm{hERG} \mathrm{K}^{+}$channel protein: Role of Hsp90 and ROS. Biochem Biophys Res Commun 2009; 388: 212-6.

52 De Ponti F, Poluzzi E, Cavalli A, Recanatini M, Montanaro N. Safety of non-antiarrhythmi drugs that prolong the QT interval or induce torsade de pointes. Drug Saf 2002; 25: 263-86.

53 Li BX, Yang BF, Zhou J, Xu CQ, Li YR. Inhibitory effects of berberine on IK1, IK, and HERG chanels of cardiac myocytes. Acta Pharmacol Sin 2001; 22: 125-31.

54 Kamiya K, Niwa R, Mitcheson JS, Sanguinetti MC. Molecular determinants of HERG channel block. Mol Pharmacol 2006; 69: 1709-16.

55 Yang BF, Xu DH, Xu CQ, Li Z, Du ZM, Wang HZ, et al. Inactivation gating determines drug potency: a common mechanism for drug blockade of HERG channels. Acta Pharmacol Sin 2004; 25: 554-60.

56 Windisch A, Timin E, Schwarz T, Stork-Riedler D, Erker T, Ecker G, et al. Trapping and dissociation of propafenone derivatives in HERG channels. Br J Pharmacol 2011; 162: 1542-52.

57 Fernadez D, Ghanta A, Kauffman GW, Sanguinetti MC. Physiocochemical features of the HERG channel drug binding site. J Biol Chem 2004; 279: 10120-7.

58 Knape K, Linder T, Wolschann P, Beyer A, Stary-Weinzinger A. In silico analysis of conformational changes induced by mutation of aromatic binding residues: consequences for drug binding in the hERG $\mathrm{K}^{+}$ Channel. PLoS One 2011; 6: e28778.

59 Moorthy NS, Ramos MJ, Fernandes PA. Human ether-a-go-go-related gene channel blockers and its structural analysis for drug design. Curr Drug Targets 2013; 14: 102-13.

60 Shan HL, Zhang Y, Bai YL, Chen X, Fan YH, Yang LL, et al. Upregulation of microRNA-1 and microRNA-133 contributes to arsneic-induced cardiac electrical remodeling. Int J Cardio 201; 167: 2789-805.

61 Varkevisser R, Houtman MJ, Linder T, de Git KC, Beekman HD, Tidwell $\mathrm{RR}$, et al. Structure-activity relationships of pentamidine-affected ion channel trafficking and dofetilide mediated rescue. $\mathrm{Br} J$ Pharmacol 2013; 169: 1322-34.

62 Rajamani S, Eckhardt LL, Valdivia CR, Klemens CA, Gillman BM, Anderson $\mathrm{CL}$, et al. Drug-induced long QT syndrome: $\mathrm{hERG} \mathrm{K} \mathrm{K}^{+}$channel block and disruption of protein trafficking by fluoxetine and norfluoxetine. Br J Pharmacol 2006; 149: 481-9.

63 Dennis AT, Nassal D, Deschenes I, Thomas D, Ficker E. Antidepressant-induced ubiquitination and degradation of the cardiac potassium channel hERG. J Biol Chem 2011; 286: 34413-25.

64 Splawski I, Shen J, Timothy KW, Lehmann MH, Priori S, Robinson JL, et al. Spectrum of mutations in long-QT syndrome genes: KVLQT1, HERG, SCN5A, KCNE1, and KCNE2. Circulation 2000; 102: 1178-85.

65 Kapplinger JD, Tester DJ, Salisbury BA, Carr JL, Harris-Kerr C, Pollevick GD, et al. Spectrum and prevalence of mutations from the first 2500 consecutive unrelated patients referred for the FAMILION Iong QT syndrome genetic test. Heart Rhythm 2009; 6: 1297-303.

66 Sanguinetti MC. HERG1 channelopathies. Pflugers Arch 2010; 460: 265-76.

67 Gong Q, Keeney DR, Molinari M, Zhou Z. Degradation of traffickingdefective long QT syndrome type II mutant channels by the ubiquitinproteasome pathway. J Bio Chem 2005; 280: 19419-25.

68 Keller SH, Platoshyn O, Yuan JX. Long QT syndrome-associated I593R mutation in HERG potassium channel activates ER stress pathways. Cell Biochem Biophys 2005; 43: 365-77.

69 Harley CA, Jesus CS, Carvalho R, Brito RM, Morais-Cabral JH. Changes in channel trafficking and protein stability caused by LQT2 mutations in the PAS domain of the HERG channel. PLoS One 2012; 7: e32654.

70 Aidery P, Kisselbach J, Gaspar H, Baldea I, Schweizer PA, Becker $\mathrm{R}$, et al. Identification and functional characterization of the novel human ether-a-go-go-related gene (hERG) R744P mutant associated with hereditary long QT syndrome 2. Biochem Biophys Res Commun 2012; 418: 830-5.

71 Balijepalli SY, Lim E, Concannon SP, Chew CL, Holzem KE, Tester DJ, et al. Mechanism of Loss of Kv11.1 $\mathrm{K}^{+}$current in mutant T421MKv11.1-expressing rat ventricular myocytesClinical perspective interaction of trafficking and gating. Circulation 2012; 126: 280918.

72 Sun Y, Zhang P, Li X, Zhang H, Li J, Liu G, et al. A novel nonsense mutation $Y 652 X$ in the S6/pore region of human ether-go-go gene found in a long QT syndrome family. Scand Cardiovasc J 2009; 43: 81-6.

73 Gong Q, Stump MR, Zhou Z. Inhibition of nonsense-mediated mRNA decay by antisense morpholino oligonucleotides restores functional expression of hERG nonsense and frameshift mutations in long-QT syndrome. J Mol Cell Cardiol 2011; 50: 223-9.

74 Gong Q, Keeney DR, Robinson JC, Zhou Z. Defective assembly and trafficking of mutant HERG channels with $\mathrm{C}$-terminal truncations in long QT syndrome. J Mol Cell Cardiol 2004; 37: 1225-33.

75 Gong Q, Stump MR, Zhou ZF. Position of premature termination codons determines susceptibility of hERG mutations to nonsensemediated mRNA decay in long QT syndrome. Gene 2014; 539: 1907.

76 Mihic A, Chauhan VS, Gao X, Oudit GY, Tsushima RG. Trafficking defect and proteasomal degradation contribute to the phenotype of a novel KCNH2 long QT syndrome mutation. PLoS One 2011; 6: e18273.

77 Stump MR, Gong Q, Zhou Z. Multiple splicing defects caused by hERG splice site mutation 2592+1G>A associated with long QT syndrome. Am J Physiol Heart Circ Physiol 2011; 300: H312-8.

78 Anson BD, Ackerman MJ, Tester DJ, Will ML, Delisle BP, Anderson $\mathrm{CL}$, et al. Molecular and functional characterization of common 
polymorphisms in HERG (KCNH2) potassium channels. Am J Physiol Heart Circ Physiol 2004; 286: H2434-41.

79 Crotti L, Lundquist AL, Insolia R, Pedrazzini M, Ferrandi C, De Ferrari $\mathrm{GM}$, et al. KCNH2-K897T is a genetic modifier of latent congenital long-QT syndrome. Circulation 2005; 112: 1251-8.

80 Yang P, Kanki H, Drolet B, Yang T, Wei J, Viswanathan PC, et al. Allelic variants in long-QT disease genes in patients with drug-associated torsades de pointes. Circulation 2002; 105: 1943-8.

81 Hayashi K, Shimizu M, Ino H, Yamaguchi M, Terai H, Hoshi N, et al. Probucol aggravates long QT syndrome associated with a novel missense mutation M124T in the N-terminus of HERG. Clin Sci 2004; 107: 175-82.

82 He FZ, McLeod HL, Zhang W. Current pharmacogenomic studies on hERG potassium channels. Trends Mol Med 2013; 19: 227-38.

83 Zeng H, Lozinskaya IM, Lin Z, Willette RN, Brooks DP, Xu X. Mallotoxin is a novel human ether-a-go-go-related gene ( $h E R G$ ) potassium channel activator. J Pharmacol Exp Ther 2006; 319: 957-62.

84 Zhou P, Babcock J, Liu L, Liu LQ, Li M, Gao ZB. Activation of human ether-a-go-go related gene ( $h E R G$ ) potassium channels by small molecules. Acta Pharmaco Sin 2011; 32: 781-8.

85 Zhang Y, Du J, Zhang Y, Sun HL, Pan ZW, Lu YJ, et al. Effects of matrine, oxymatrine and resveratrol on HERG channel expression. Yao Xue Xue Bao 2007; 42: 139-44.

86 Wang TZ, Andrew HC, Kang YD, Cui Z, Guo J, Yang TH, et al. Muscarinic receptor activation increases hERG channel expression through phosphorylation of ubiquitin ligase Nedd4-2. Mol Pharmacol 2014; 85: 877-86.

87 Mehta A, Ssequiera GL, Ramachandra CJ, Sudibyo Y, Chung YY, Sheng JW, et al. Re-trafficking of hERG reverses long QT syndrome 2 phenotype in human iPS-derived cardiomyocytes. Cardiovasc Res 2014; 102: 497-506.

88 Casis O, Olesen SP, Sanguinetii MC. Mechanism of action of a novel human ether-a-go-go-related gene channel activator. Mol Pharmacol
2006; 69: 658-65.

89 Asayama M, Kurokawa J, Shirakawa K, Okuyama H, Kagawa T, Okada $\mathrm{J}$, et al. Effects of an hERG activator, ICA-105574, on electrophysiological properties of canine hearts. J Pharmacol Sci 2013; 121: 1-8.

90 Diness TG, Yeh YH, Qi XY, Chartier D, Tsuji Y, Hansen RS, et al. Antiarrhythmic properties of a rapid delayed-rectifier current activator in rabbit models of acquired long QT syndrome. Cardiovasc Res 2008; 79: 61-9.

91 Mao HY, Lu XL, Karush JM, Huang XY, Yang X, Ba YN, et al. Pharmacologic approach to defective protein trafficking in the E637K-hERG mutant with PD-118057 and thapsigargin. PLoS One 2013; 8: e65481.

92 Rajamani S, Anderson CL, Anson BD, January CT. Pharmacological rescue of human $\mathrm{K}^{+}$channel long-QT2 mutations human ether-a-gogo-related gene rescue without block. Circulation 2002; 105: 28305.

93 Lu XL, Yang X, Huang X, Huang C, Sun HH, Jin L, et al. RNA interference targeting E637K mutation rescues the hERG channel currents and restores its kinetic properties. Heart Rhythm 2013; 10: 128-36.

94 Matsa E, Dixon JE, Medway C, Georgiou O, Patel MJ, Morgan K, et al. Allele-specific RNA interference rescues the long-QT syndrome phenotype in human-induced pluripotency stem cell cardiomyocytes. Eur Heart J 2014; 35: 1078-87.

95 Thorrez L, Sampaolesi M. The future of induced pluripotent stem cells for cardiac therapy and drug development. Curr Pharm Des 2011; 17 : 3258-70.

96 Sampson HM, Lam H, Chen PC, Zhang D, Mottillo C, Mirza M, et al. Compounds that correct F508del-CFTR trafficking can also correct other protein trafficking diseases: an in vitro study using cell lines. Orphanet J Rare Dis 2013; 8: 11.

97 Dong DL, Li Z, Wang HZ, Du ZM, Song WH, Yang BF. Acidification alters antiarrhythmic drug blockade of the ether-a-go-go-related gene (HERG) channels. Basic Clin Pharmacol Toxicol 2004; 94: 209-12. 